Article

\title{
Structural Basis for Broad Substrate Selectivity of Alcohol Dehydrogenase YjgB from Escherichia coli
}

\author{
Giang Thu Nguyen ${ }^{1}$, Yeon-Gil Kim ${ }^{2}$, Jae-Woo Ahn ${ }^{3, *}$ and Jeong Ho Chang $1,4, *(\mathbb{D})$ \\ 1 Department of Biology Education, Kyungpook National University, 80 Daehak-ro, Buk-gu, \\ Daegu 41566, Korea; thugiang1995@gmail.com \\ 2 Beamline Science Division, Pohang Accelerator Laboratory, 127 Jigok-ro, Nam-Gu, Pohang, \\ Gyoungbuk 37673, Korea; ygkim76@postech.ac.kr \\ 3 Postech Biotech Center, Pohang University of Science and Technology, 77 Cheongam-ro, Nam-Gu, Pohang, \\ Gyoungbuk 37673, Korea \\ 4 Department of Biomedical Convergence Science and Technology, Kyungpook National University, \\ 80 Daehak-ro, Buk-gu, Daegu 41566, Korea \\ * Correspondence: jaewooahn@postech.ac.kr (J.-W.A.); jhcbio@knu.ac.kr (J.H.C.); \\ Tel.: +82-54-279-8648 (J.-W.A.); +82-53-950-5913 (J.H.C.); Fax: +82-54-279-8379 (J.-W.A.); \\ +82-53-950-6809 (J.H.C.); M.P.: +82-10-9578-1734 (J.-W.A.); +82-10-4765-1107 (J.H.C.)
}

Academic Editor: Hyuma Masu

Received: 8 April 2020; Accepted: 19 May 2020; Published: 21 May 2020

\begin{abstract}
In metabolic engineering and synthetic biology fields, there have been efforts to produce variable bioalcohol fuels, such as isobutanol and 2-phenylethanol, in order to meet industrial demands. YjgB is an aldehyde dehydrogenase from Escherichia coli that shows nicotinamide adenine dinucleotide phosphate (NADP)-dependent broad selectivity for aldehyde derivatives with an aromatic ring or small aliphatic chain. This could contribute to the design of industrial synthetic pathways. We determined the crystal structures of YjgB for both its apo-form and NADP-complexed form at resolutions of 1.55 and $2.00 \AA$, respectively, in order to understand the mechanism of broad substrate selectivity. The hydrophobic pocket of the active site and the nicotinamide ring of NADP(H) are both involved in conferring its broad specificity toward aldehyde substrates. In addition, based on docking-simulation data, we inferred that $\pi-\pi$ stacking between substrates and aromatic side chains might play a crucial role in recognizing substrates. Our structural analysis of YjgB might provide insights into establishing frameworks to understand its broad substrate specificity and develop engineered enzymes for industrial biofuel synthesis.
\end{abstract}

Keywords: YjgB; aldehyde dehydrogenase; broad specificity; crystal structure; hydrophobic pocket; docking-simulation

\section{Introduction}

The production of large yields of bio-renewable chemicals and biofuels from biomass is a primary goal in the fields of metabolic engineering and synthetic biology. The production of butanol, isobutanol, propanediol, butanediol, and aromatic alcohols while using designed synthetic pathways of microorganisms has been attempted to meet industrial demands [1-6]. In the synthetic pathway of these alcohols, the final step is commonly the reduction of an aldehyde through alcohol dehydrogenases. Escherichia coli possess strong alcohol dehydrogenases that could contribute to producing alcohols through a designed pathway [7]. These powerful enzymes, including YjgB, YahK, and YqhD, show nicotinamide adenine dinucleotide phosphate (NADP)-dependent activities for a variety of aldehydes. YqhD has 17\% sequence identity with YjgB and it shows reductase activity for broad short-chain aldehydes, including butyraldehyde, for which it shows the strongest activity, as well 
as glyceraldehyde, malondialdehyde, isobutyraldehyde, methylglyoxal, propanealdehyde, acrolein, furfural, glyoxal, 3-hydroxypropionaldehyde, glycolaldehyde, acetaldehyde, and acetol [8]. Unlike YqhD, YjgB, and YahK, which have a similarity of 35\% sequence identity, strongly prefer benzaldehyde and furfural. In terms of turnover number, hexanal is supposed to be the best substrate for both enzymes. However, no activity has been measured when using a ketone as a substrate [7]. YjgB and YahK have been utilized in pathway design for the production of aromatic compounds for 2-(4-hydroxyphenyl)ethanol and 4-hydroxyphenyllactic acid. In addition, YjgB has recently been reported to produce 2-phenylethanol (2-PE) and 2-phenylethyacetate (2-PEAc) in E. coli-based metabolic engineering $[9,10]$. These 2-PE and 2-PEAc are fragrance compounds containing high-value flavor with a wide range of applications in the cosmetic, perfumery, and food industries. Although the chemical synthesis of 2-PE and 2-PEAc has advantage in cost, many consumers prefer more natural or bio-products in the industry of flavor $[10,11]$. On the other hand, natural extracts obtained from plant sources have high-cost, due to their low extraction yields [12,13].

Structurally, YjgB, together with YahK and YqhD, are grouped in the medium-chain dehydrogenase/reductase superfamily. YqhD belongs to the family III metal-dependent polyol dehydrogenases, while YjgB and YahK are classified as members of the cinnamyl alcohol dehydrogenase family $[14,15]$. Generally, several medium-chain alcohol dehydrogenases have been revealed to exist in bacteria as dimers or tetramers, while using $\mathrm{NAD}(\mathrm{H})$ or $\mathrm{NADP}(\mathrm{H})$ as a cofactor and one catalytic zinc ion at the active site. In some dimeric alcohol dehydrogenases, an additional zinc might be required to further support the stability of the external loop structures [16]. Like other Zn-containing members in the family, $\mathrm{YjgB}$ and YahK both possess the $\mathrm{GHEX}_{2} \mathrm{GX}_{5}(\mathrm{G}, \mathrm{A}) \mathrm{X}_{2}(\mathrm{I}, \mathrm{V}, \mathrm{A}, \mathrm{C}, \mathrm{S})$ motif and the $\mathrm{GX}_{1-3} \mathrm{GX}_{1-3} \mathrm{G}$ pattern located in the nucleotide-binding region [7]. Although Sulzenbacher et al. comprehensively studied the YqhD structure [17], little information is available regarding the other two versatile enzymes, except for the YahK structure, which is deposited in the Protein Data Bank (PDB code: 1UUF) without an attached report. To fill this gap, this study aims to determine the YjgB apo and complex structures of E. coli and compare them with the structures of YahK and YqhD to indicate the substrate specificity of YjgB. The findings of this study are useful to the field of protein engineering, as they inform the better production of 2-PE and 2-PEAc.

\section{Results}

\subsection{Overall Structure}

The E. coli YjgB ( $E c Y j g B)$ was crystallized in the monoclinic space group, $C 2$, and its crystal structure was successfully determined at $1.55 \AA$ resolution (Table 1). As predicted from sequence alignment (Figure 1), the $E c Y j g B$ structure was found to belong to a typical cinnamyl alcohol dehydrogenase (CAD) family [17] and sinapyl alcohol dehydrogenase (SAD) family [17], which consists of a nucleotide binding domain (NBD, residues 165-286) and a substrate-binding domain (SBD, residues 1-164 and 287-338) (Figure 2a). The NBD (nucleotide-binding domain) was shown to adopt a canonical Rossmann fold [18] that was composed of alternating beta strands and alpha helixes, which can hold cofactor $\mathrm{NAD}(\mathrm{H}) / \mathrm{NADP}(\mathrm{H})$. As in other $\mathrm{Zn}$-dependent CAD and SAD families, PDBePISA (Proteins, Interfaces, Structures, and Assemblies) analysis showed that this Rossmann fold can form a dimer [19]. The structure of the dimeric interface showed that dimerization region I (residues 268-270) and II (residues 280-284) establish the dimerization interface by generating two $\beta$-sheets (Figure 2b). These dimerizations are involved in forming a hydrophobic active site that consists of residues with aromatic rings (see 2.2 for more detail). 
Table 1. Data collection, phasing, and refinement statistics for YjgB from E. coli.

\begin{tabular}{|c|c|c|}
\hline & Apo & PEG-NADP Complex \\
\hline \multicolumn{3}{|l|}{ Data collection } \\
\hline Wavelength $(\AA)$ & 0.97934 & 1.00003 \\
\hline Space group & C2 & $\mathrm{C} 222_{1}$ \\
\hline \multicolumn{3}{|l|}{ Cell dimensions } \\
\hline$a, b, c(\AA)$ & $133.14,64.47,81.66$ & $64.97,138.99,168.51$ \\
\hline$\alpha, \beta, \gamma\left({ }^{\circ}\right)$ & $90.00,106.14,90.00$ & $90.00,90.00,90.00$ \\
\hline Resolution $(\AA)$ & $50.00-1.55(1.58-1.55)^{\mathrm{a}}$ & $30.00-2.00(2.06-2.00)$ \\
\hline$R_{\text {merge }}(\%){ }^{b}$ & $7.2(57.3)$ & $10.9(59.2)$ \\
\hline$I / \sigma(I)$ & $36.2(3.8)$ & $19.2(5.3)$ \\
\hline Unique reflection & $95566(4760)$ & $51912(4358)$ \\
\hline Completeness (\%) & $100.0(100.0)$ & $99.9(100.0)$ \\
\hline Redundancy & $7.4(7.4)$ & $13.8(14.1)$ \\
\hline $\mathrm{CC}_{1 / 2}$ & $97.5(89.4)$ & $99.9(96.5)$ \\
\hline \multicolumn{3}{|l|}{ Refinement } \\
\hline Resolution $(\AA)$ & $25.02-1.55$ & $29.55-2.00$ \\
\hline No. reflections & 95547 & 99594 \\
\hline$R_{\text {work }}(\%)^{\mathrm{c}} / R_{\text {free }}(\%)^{\mathrm{d}}$ & $16.1 / 19.4$ & $16.0 / 20.0$ \\
\hline No. atoms & 6093 & 5493 \\
\hline $\mathrm{Zn}$ & 4 & 4 \\
\hline Glycerol & 36 & 12 \\
\hline $\mathrm{NO}_{3}$ & 8 & \\
\hline NADP & & 96 \\
\hline PEG & & 14 \\
\hline Water & 790 & 591 \\
\hline Averaged $B$-factors & 24.8 & 27.9 \\
\hline \multicolumn{3}{|c|}{ R.m.s. deviations from ideal value } \\
\hline Bond lengths $(\AA)$ & 0.013 & 0.008 \\
\hline Bond angles $\left({ }^{\circ}\right)$ & 1.400 & 0.903 \\
\hline PDB ID & 7BU2 & 7BU3 \\
\hline
\end{tabular}

${ }^{\mathrm{a}}$ Values in parentheses are for highest-resolution shell. ${ }^{\mathrm{b}} R_{\text {merge }}=\sum_{h k l} \sum_{i}\left|I_{i}(h k l)-<I(h k l)>V\right| \sum_{h k l} \sum_{i} I_{i}(h k l)$ where $\left\langle I(h k l)>\right.$ is the average intensity of the $i$-th observations. ${ }^{\mathrm{c}} R_{\text {work }}=\sum_{h k l} \| F_{\text {obs }}(h k l)|-| F_{\text {calc }}(h k l)|| \sum_{h k l}\left|F_{\text {obs }}(h k l)\right| . R_{\text {work }}$ is calculated with $95 \%$ of reflections used for structure refinement. ${ }^{d} R_{\text {free }}$ is calculated for the remaining $5 \%$ of reflections randomly selected and excluded from refinement.

As the $\mathrm{GX}_{1-3} \mathrm{GX}_{1-3} \mathrm{G}, \mathrm{GHEX}_{2} \mathrm{GX}_{5}(\mathrm{G} / \mathrm{A}) \mathrm{X}_{2}(\mathrm{I} / \mathrm{V} / \mathrm{A} / \mathrm{C} / \mathrm{S})$ motif, and catalytic $\mathrm{Zn}^{2+}$ ion coordination sites, Cys41, His63, and Cys152, are conserved in sequence alignment (Figure 1), they play a role in coordinating $\mathrm{Zn}^{2+}$ ions and generating a NAD/NADP binding site. The electron density of NADP is clearly shown in the NADP $(\mathrm{H})$ binding site of the Ec YjgB-NADP complex structure (Figure 2c). We also found that the NADP was well overlaid with NADP(H) molecules of CAD/SAD structures (data not shown). We observed that His42, Ser43, Ser46, Gly179, Leu180, Ser199, Ser200, Asn201, Glu208, Ser285, Gly327, and Arg332 constitute the NADP(H) binding site. In addition, conserved Ser199 and Lys204 (Figure 2c) make it possible to specifically select the $2^{\prime}$ phosphate of NADP(H) as a stabilizing $2^{\prime}$ phosphate of the dinucleotide while using a hydrogen bond and charge-charge interaction. 

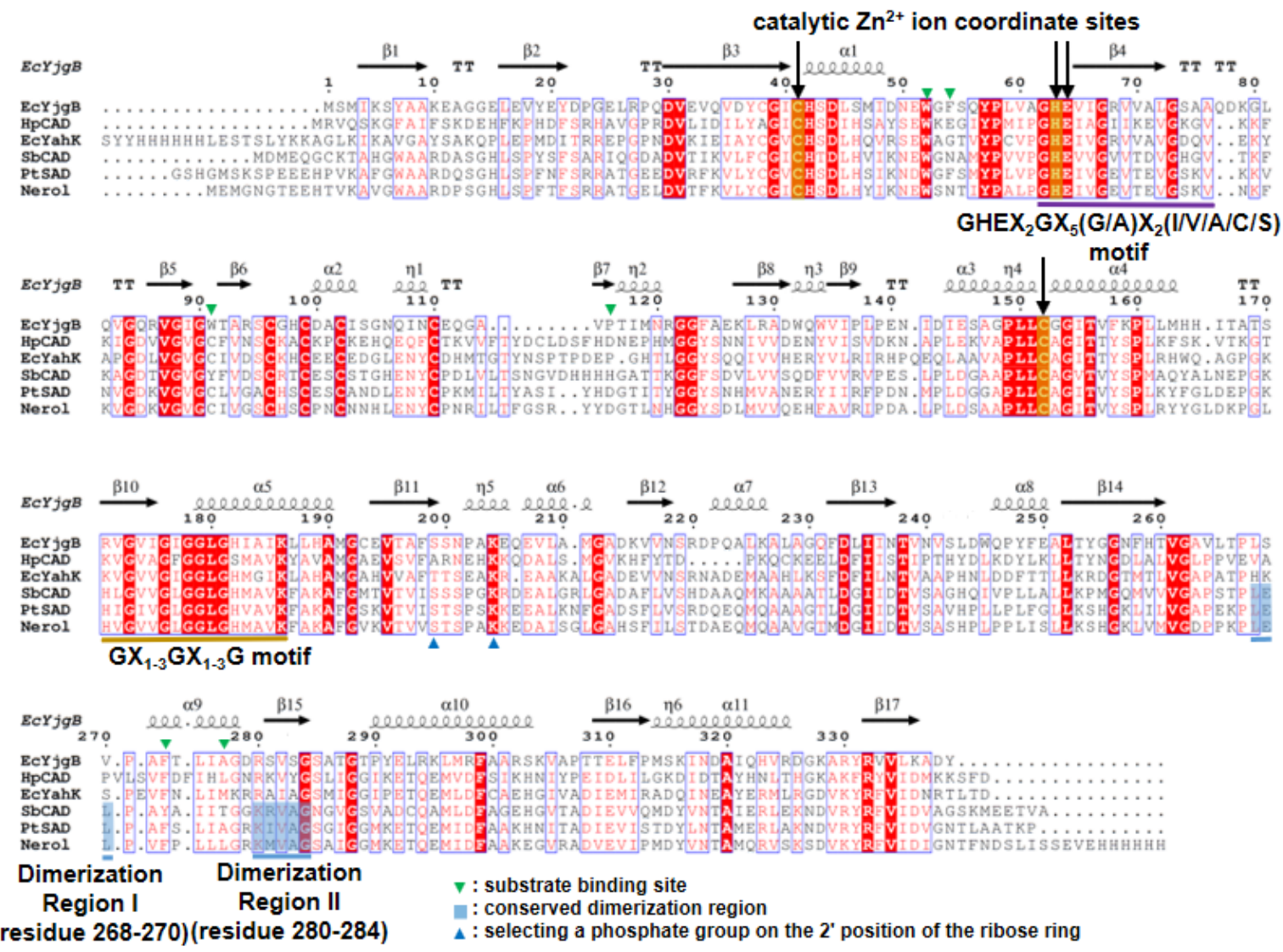

Figure 1. Sequence alignment for $E c Y j g B, H p C A D, E c Y a h K, S b C A D, P t C A D$, and Nerol. Sequences of EcYjgB, Escherichia coli BL21 (DE3) YjgB (B21_04099); HpCAD, Helicobacter pylori cinnamyl alcohol dehydrogenase (PDB code: 3TWO); Ec YakK, Escherichia coli BL21 (DE3) YahK (PDB code: 1UUF); SbCAD, Sorghum bicolor cinnamyl alcohol dehydrogenase (SbCAD) (PDB code: 5VKT); PtSAD, Populus tremuloides SAD (sinapyl alcohol dehydrogenase) (PDB code: 1YQD); Nerol, Yersinia pseudotubercluosis IP32953 nerol dehydrogenase (PDB code: 5Z0C) are aligned. Sequence identities for $E c Y j g B$ are $30.4 \%$ (HpCAD), 35.9\% (EcYakK), 35.0\% (SbCAD), 35.9\% (PtSAD), and 34.7\% (Nerol), respectively. Down arrows, and purple and golden under-bars indicate catalytic $\mathrm{Zn}^{2+}$ ion coordinate sites, $\mathrm{GHEX}_{2} \mathrm{GX}_{5}(\mathrm{G} / \mathrm{A}) \mathrm{X}_{2}(\mathrm{I} / \mathrm{V} / \mathrm{A} / \mathrm{C} / \mathrm{S})$, and $\mathrm{GX}_{1-3} \mathrm{GX}_{1-3} \mathrm{G}$ motif, respectively. Green downside and blue upside triangles indicate substrate-binding sites and the amino acids important for selecting the phosphate group on the 2 ' position of the ribose ring in nicotinamide adenine dinucleotide phosphate (NADP). Sky blue boxes represent conserved dimerization region. This multiple sequence alignment was performed using Clustal/W and prepared with the ESPript software package [20,21]. 

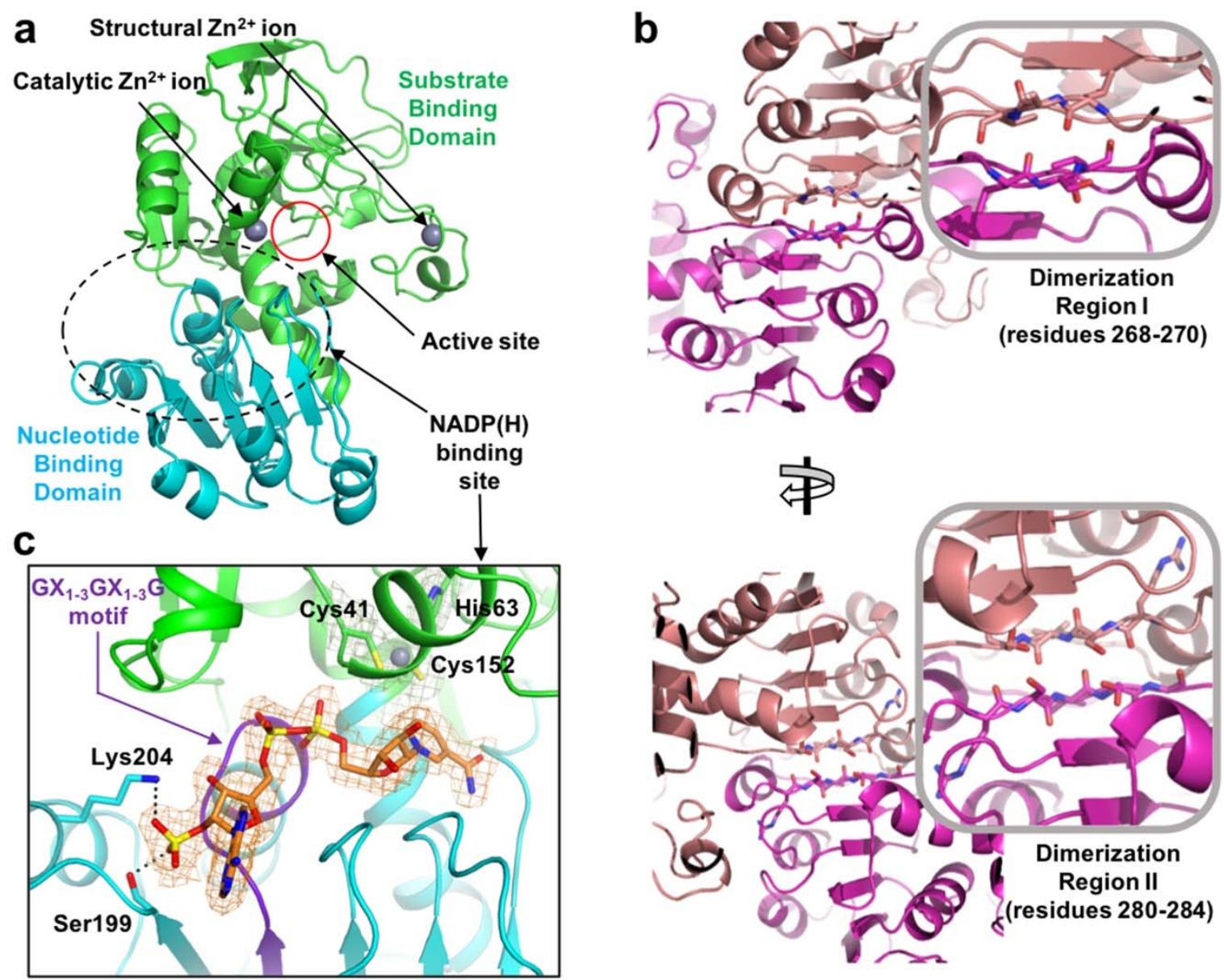

Figure 2. Structure of Escherichia coli YjgB (EcYjgB). (a) Overall structure. (b) Dimeric interface of EcYjgB. In the outcome of the Proteins, Interfaces, Structures, and Assemblies (PISA) analysis, the dimeric chains are colored as salmon and purple [19] (c) NADP binding site. The SBD (substrate-binding domain) and NBD (nucleotide-binding domain) are represented as green and cyan, respectively. The $2 F o-F c$ electron densities of NADP (orange), and zinc ion (grey) with its coordinated residues (green) are shown at the $1.0 \sigma$ contoured level with their corresponded colors. The $\mathrm{GX}_{1-3} \mathrm{GX}_{1-3} \mathrm{G}$ motif is indicated as a purple cartoon.

\subsection{Zinc Coordination and Active Site}

We observed a cleft between the SBD (substrate-binding domain) and NBD (nucleotide-binding domain) that forms the active site with a catalytic $\mathrm{Zn}^{2+}$ ion (Figure 2a). It was found that the catalytic $\mathrm{Zn}^{2+}$ ion is coordinated by the tetrahedral combination of Cys41, His63, Glu64, and Cys152, which were well conserved (Figure 1). In the apo structure, distances ranging from 2.0 to $2.3 \AA$ were shown between

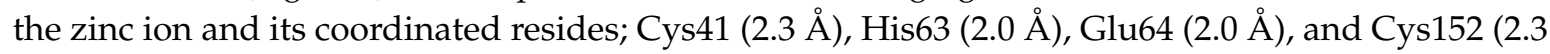
$\AA$ ) (Figure 3a). Meanwhile, each position of the four residues was $0.6 \AA$ farther away from catalytic zinc in the EcYjgB-NADP complex, based on its superimposed structure with the apo form through the NBD region (Figure $3 b$ ). Although the catalytic components were relatively relocated toward the nicotinamide ring of NADP, their positions were still not properly matched to those of other CAD/SAD structures. 
a
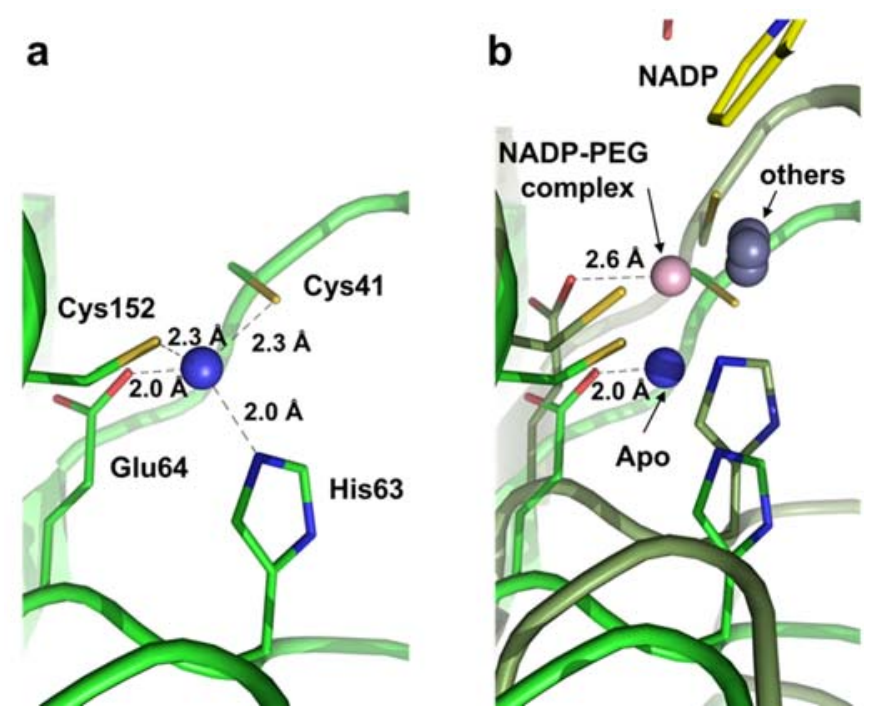

Figure 3. Catalytic zinc coordination. (a) Catalytic zinc coordination and its coordinated residues in the apo structure. The blue sphere indicates a zinc ion of EcYjgB. (b) Catalytic zinc coordination of superposed apo and NADP complex structures. The blue and light-pink spheres indicate the catalytic zinc ions of apo and NADP complex, respectively. The apo and NADP complex structures are drawn by green and dark-green cartoons, respectively.

Interestingly, in the EcYjgB-NADP complex structure, we found an electron density of polyethylene glycol (PEG) density in the vicinity of the active site (see Section 2.3). It seemed that PEG did not induce the zinc relocation by dissociation of the glutamate residue since PEG does not contain an aldehyde group, as observed in the structures of SbCAD (PDB code: 5VKT) [17], yeast alcohol dehydrogenase (PDB code: 4W6Z) [22], and E.coli ethanol inducible dehydrogenase (PDB code: 4GKV) [23]. Consequently, the catalytic zinc could not go further toward a substrate as well as generate a zinc-bound alkoxide ion, which might cause insufficient relocation of catalytic zinc in the EcYjgB-NADP complex structure.

\subsection{Substrate-Binding Pocket by Conformational Change}

We tried to obtain the crystal structure with the substrate or NADP to investigate the reaction mechanism of EcYjgB for broad substrates. During the determination of the structure comprising NADP, we found that a polyethylene glycol (PEG) fragment occupied its catalytic cavity, which was quite similar to a hexanaldehyde (Figure 4a). As it is holding the NADP, the SBD moves about $4.0 \AA$ toward the NBD with a root-mean-square deviation (r.m.s.d.) of $1.02 \AA$ when compared to the apo structure (Figure $4 \mathrm{~b}$ ). It represents that NADP binding can generate the closed form, as observed in other CAD/SAD family. The superposition of the EcYjgB-NADP complex structure with a closed form of SbCAD implied that NADP binding is likely to induce the conformational change upon substrate-binding to hold a substrate or a PEG fragment (Figure 4c). Thus, several conserved catalytic residues were relocated to the vicinity of their counterpart in SbCAD, such as Ser42, Trp52, Trp91, and Tyr95. The tryptophan residues may play a crucial role in holding PEG due to their aromatic rings. The catalytic zinc of NADP complex was not completely moved to the proper position, although PEG was occupied at the substrate-binding pocket, as mentioned previously. This might indicate that the absence of aldehyde group in PEG led positions of Cys41, Ser42, and Glu64 adjacent to those of counterparts in SbCAD. Interestingly, Phe273' of dimeric chain was located unalike to the closed form of $S b C A D$ (Figure 4c). In contrast, Tyr288' faced outside of the substrate-binding pocket without an aromatic ring or its equivalent to that in the formic acid. Phe $273^{\prime}$ turns toward the substrate binding site since PEG occupies the substrate-binding pocket after the conformational change upon NADP 
binding. Consequently, the oxygen from PEG nearby Phe273' could form a kind of anion- $\pi$ interaction that contributes to holding the ligand.

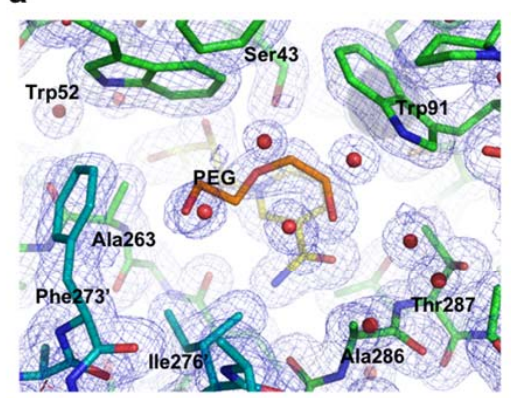

b

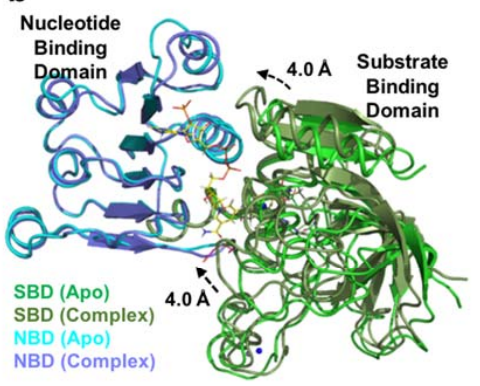

c

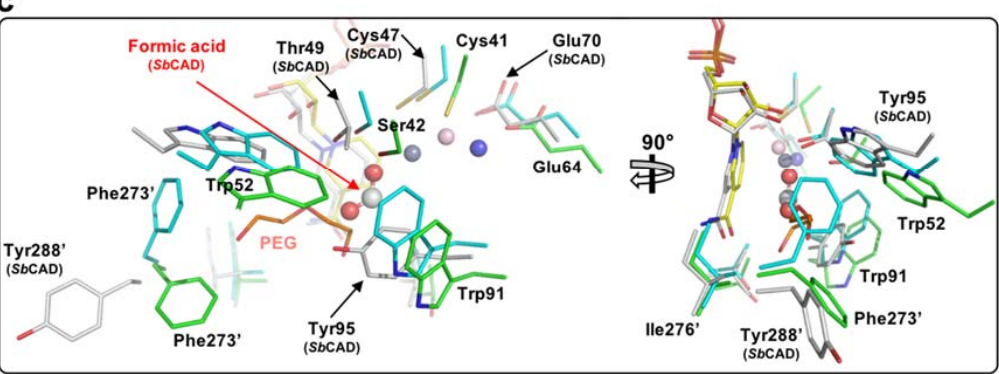

d
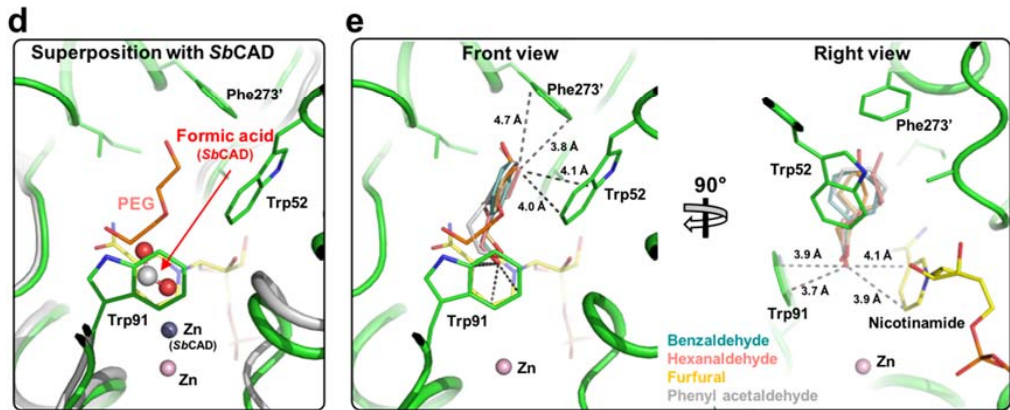

Figure 4. Active site and Docking simulation. (a) Electron density map of the active site in the EcYjgB-NADP-polyethylene glycol (PEG) complex structure. The 2Fo-Fc densities including PEG are shown at the $1.0 \sigma$ contoured level with a dark-blue colored mesh. The monomer and its symmetry related molecule are colored in green and cyan, respectively. (b) Conformational difference between the apo and complex structure. The SBD (substrate-binding domain) of the apo and complex structures and NBD (nucleotide-binding domain) of the apo and complex structures are colored as green, dark green, cyan, and light purple, respectively. (c) Conformational change in the active site. Apo, NADP complex, and closed form of SbCAD (PDB code: 5VKT) are colored in green, cyan, and gray colors, respectively. Formic acid in SbCAD is represented as a ball and stick with gray-colored carbon and red-colored oxygen. Zinc ions of apo-YjgB, EcYjgB-NADP, and closed form of SbCAD are represented as light-blue, light-pink, and silver spheres, respectively. PEG in the EcYjgB-NADP structure are drawn as stick with orange carbon and red oxygen. (d) Docking-simulation and comparison with PEG and formic acid of SbCAD. EcYjgB-NADP structure and SbCAD are colored in green and silver, respectively. Formic acid in SbCAD is represented as corresponded to Figure 4c. Zinc ions of EcYjgB-NADP, and closed form of $S b C A D$ are shown in light-pink and gray spheres, respectively. PEG in the EcYjgB-NADP structure are drawn as stick with orange-carbon and red-oxygen. (e) Two side views of active site in the EcYjgB-NADP complex structure. Overlaid ligands calculated from the docking simulation are shown as indicated colors. Dashed lines represent the acceptable distances in $\pi-\pi$ stacking. Light-pink sphere indicates the catalytic zinc of EcYjgB-NADP complex. 


\subsection{Substrate Specificity}

The PEG occupancy could present the substrate-binding site reasonable and acceptable. The conformational change and the dimerization result in the substrate-binding site. The substrate-binding site was found to consist of Ser43, Trp52, Trp91, Thr287 from NBD, Ala263 and Ala286 of SBD, Phe273', and Ile276' from the other dimeric chain (Figure 4a). The combination of Trp52, Trp91, Phe273', and Ile276' was observed to constitute the analog of the hydrophobic cavity with appropriate hydrophobicity, polarity, and size for the substrate. Trp52 and Trp91 were shown to hold the ligand through $\mathrm{CH}-\pi$ stacking, which appeared to be a primary contributor for substrate recognition. Particularly, turning Phe273' toward the substrate-binding pocket possibly forms a kind of anion- $\pi$ interaction. Catalytic zinc-coordinating residues are similarly positioned to the closed form, despite their insufficient zinc location. Thus, we determined that the conformation of PEG and its interactions with catalytic residues are largely similar to those of authentic substrate.

We carried out docking-simulations while using Autodock Vina software in order to suggest the substrate-binding mode and critical residue for substrate binding [24]. a EcYjgB protein that was derived from the EcYjgB-NADP-PEG complex was used as a target molecule, because it could tightly bind substrates in the active site, which was constituted by proper arrangement of NADP and PEG. Hexanaldehyde, benzaldehyde, furfural, and phenylacetaldehyde, known as aldehyde substrates, were used for the docking simulation $[7,9,10]$. The highest calculated binding affinities were -5.7 , $-4.6,-4.3$, and -6.1 , respectively, for benzaldehyde, hexanaldehyde, furfural, and phenylacetaldehyde. Their calculated binding modes were somewhat comparable to the formic acid of SbCAD as well as the PEG fragment of the NADP complex structure (Figure 4d). From the predicted binding mode, we could figure out the Trp52 function to hold a substrate with an aromatic ring or $\mathrm{CH}$ chain due to $\pi-\pi$ stacking interactions. Trp52 is not perfectly stacked to substrates and it is found Parallel-Displaced $\pi-\pi$ stacking to aromatic rings and $\mathrm{CH}$ chains, at distance of below $\sim 4.1 \AA$ (Figure $4 \mathrm{e}$ ). In addition, Phe273' of the dimeric chain seems to limit the size of substrates by forming T-shaped $\pi-\pi$ stacking, despite not being complete vertically with distances of 3.8-4.7 $\AA$. Finally, the oxygen of aldehyde from substrates are located between Trp91 and the nicotinamide ring of NADP (Figure 4e). They are regarded as perpendicular anion- $\pi$ stackings with the distances of 3.7-4.1 $\AA$. Therefore, it is acceptable to infer that these $\pi-\pi$ stackings by aromatic side chain in active site play a critical role in holding substrates and conferring the broad selectivity.

\section{Discussion}

The final process is the reduction of an aldehyde. E. coli has strong aldehyde dehydrogenases with a broad specificity to bio-synthesize small size alcohols, such as butanol, isobutanol, propanediol, butanediol, 2-PE, and 2-PEAc. We determined the structure of YjgB from E. coli to understand this broad specificity and contribute to designing more efficient pathways for alcohol production. From this structure, we suggested the mechanism of broad substrate specificity. The active site was found to be constituted by the combination of isoleucine, alanine, tryptophan, phenylalanine, and the nicotinamide ring of NADP, which enable the broad specificity for small hydrophobic aldehydes with aromatic rings and short $\mathrm{CH}$ chains. This broad specificity of aldehyde dehydrogenases in E. coli might assist cell survival under oxidative stress [7]. It is known that aldehydes are very reactive and toxic to cells, since the aldehyde group $(\mathrm{CHO})$ is a strong electrophile that can undergo additional reactions. Small molecular aldehydes are naturally generated in cells and produced in small amounts through the intermediary metabolism of natural chemicals. These aldehydes are primarily metabolized by dehydrogenases or glutathione-dependent pathways, and toxicity results from increased intracellular concentrations following these detoxification systems being overwhelmed [25]. Bacteria also encounter oxidative stresses: reactive oxygen species (ROS) are generated from a number of sources, including the leakage of single electrons from respiratory enzymes and environmental stresses, such as UV radiation [26]. Broad specificity helps the resistance of $E$. coli to small aldehydes with aromatic rings and $\mathrm{CH}$ chains. This broadness appears to confer more competitiveness and flexibility for a limited gene pool of bacteria. 
In comparison of the substrate docking-simulation data to the NADP complex structure incorporating a PEG fragment (Figure 4d,e), the aldehyde groups on the substrates that are located toward the catalytic zinc are in opposition to the aldehyde out-group of the PEG complex structure. Despite the proper directions of aldehyde groups, their distances to the catalytic zinc are still insufficient to make zinc-ligated aldehyde intermediates. It is assumed that those outputs are caused by unreflecting complete induced fit in the target molecule during the docking-simulation. Therefore, the binding of an authentic substrate, not a PEG molecule, to the active site pocket leads to the formation of the activated zinc coordination and actual substrate bound conformation for catalytic reactions. The B-factors of zinc and Glu64 increased more than twice as Glu64 moved away from the zinc ion by NADP binding (Table 2). Given these, we can speculate that a substrate binds to the widened gap between Trp91 and nicotinamide ring, and the substrate can be likely located a little bit below the site of simulation. Regarding the zinc relocation that can be induced by the substrate-entry, it is expected that the zinc ion can approach the aldehyde group of the substrate to generate a zinc-bound substrate intermediate. Taken together, the NADP-PEG complex structure with multiple substrate docking simulations could contribute to understanding the substrate-binding mechanism in EcYjgB.

Table 2. B-factor comparisons for $\mathrm{Zn}$ and its coordinate residues.

\begin{tabular}{cccc}
\hline & Apo $\left(\AA^{2}\right)$ & NADP-PEG Complex $\left(\AA^{2}\right)$ & $\begin{array}{c}\text { Ratio } \\
\text { (Complex/Apo) }\end{array}$ \\
\hline Zn & 17.8 & 38.8 & 2.2 \\
Cys41 & 17.0 & 31.9 & 1.9 \\
His63 & 15.5 & 31.9 & 2.1 \\
Glu64 & 14.6 & 33.8 & 2.3 \\
Cys152 & 15.6 & 28.1 & 1.8 \\
\hline Whole protein & 24.8 & 27.9 & 1.1 \\
\hline
\end{tabular}

\section{Materials and Methods}

\subsection{Cloning, Expression, and Purification}

The $Y j g B$ gene was digested with $N d e I$ and $X h o I$ restriction enzymes and inserted into a pET30a vector (Novagen) after PCR amplification. The EcYjgB protein, containing the 6X His tag at its C-terminus, was expressed in the E. coli strain BL21 (DE3). The cells were cultured in an LB medium containing kanamycin at $37^{\circ} \mathrm{C}$ until reaching an absorbance of 0.7 at $600 \mathrm{~nm}$. Protein expression was induced by $1.0 \mathrm{mM}$ isopropyl $\beta$-D-1-thiogalactopyranoside (IPTG), and the cells were then incubated

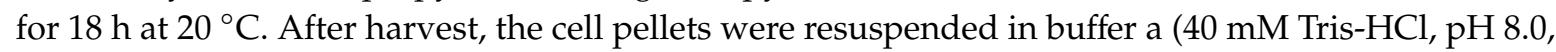
and $5 \mathrm{mM} \beta$-mercaptoethanol) and then disrupted by ultrasonication. The cell debris was removed by centrifugation at $12,000 \times g$ for 20 min., and the lysate was bound to Ni-NTA agarose (QIAGEN). After first washing with buffer a containing $20 \mathrm{mM}$ imidazole, the bound proteins were then eluted with buffer a containing $300 \mathrm{mM}$ imidazole. The EcYjgB protein was purified while using size exclusion chromatography to obtain high purity for crystallization (Superdex200, GE Healthcare, Chicago, IL, USA). The purified proteins were concentrated to $20 \mathrm{mg} / \mathrm{mL}$ in a solution with $20 \mathrm{mM}$ Tris- $\mathrm{HCl}, \mathrm{pH} 7.5$, and $5 \mathrm{mM} \beta$-mercaptoethanol.

\subsection{Crystallization}

Crystallization of the apo EcYjgB was initially performed using crystal screening kits (Hampton Research Co., Aliso Viejo, CA, USA and Emerald Biostructures Co., Bainbridge Island, WA, USA) and by using the sitting-drop vapor-diffusion method at $20^{\circ} \mathrm{C}$. Each experiment consisted of mixing $1.0 \mu \mathrm{L}$ of the protein solution with $1.0 \mu \mathrm{L}$ of reservoir solution and equilibrating this against $0.1 \mathrm{~mL}$ of the reservoir solution. Thin plate-shaped crystals were observed after 1-3 days. The diffraction of these crystals was not suitable for the determination of their structure. We adjusted the concentration of the precipitant, polyethylene glycol (PEG) 3350, and the pH of the reservoir solution to obtain high 
purity for crystallization. The suitable crystals for diffraction were obtained under conditions of $0.1 \mathrm{M}$ Tris- $\mathrm{HCl}$, pH 7.0, 16\% (w/v) PEG 3350, and 0.2 M lithium sulfate. The suitable crystals grew within 1-3 days to dimensions of approximately $200 \times 50 \times 10 \mu \mathrm{m}$. The apo EcYjgB crystals were soaked into the crystallizing buffer supplemented with $5 \mathrm{mM} \mathrm{NADP}$ for $30 \mathrm{~min}$. at $7^{\circ} \mathrm{C}$ to enable the formation of the EcYjgB-NADP complex. In this case, apo-form crystals were prepared within 1 day by hanging-drop vapor diffusion method at $20^{\circ} \mathrm{C}$, under the condition of $0.08 \mathrm{M}$ Ammonium citrate tribasic $\mathrm{pH} 7.0$ and 18\% PEG 3350.

\subsection{Data Collection and Processing}

A reservoir solution containing 30\% $(v / v)$ glycerol was used as a cryo-protectant for the crystals. The data sets were collected at $100 \mathrm{~K}$ at 7A of the Pohang Accelerator Laboratory (Pohang, Korea). The best apo and NADP-complexed crystals were diffracted to resolutions of 1.55 and $1.45 \AA$, respectively. The apo crystals belonged to the $C 2$ space group with unit cell parameters of $a=133.14 \AA, b=64.47$ $\AA, c=81.66 \AA, \alpha=\gamma=90.00^{\circ}$, and $\beta=106.14^{\circ}$, and NADP-complexed possessed $C 222_{1}$ unit cell of $a=64.97 \AA, b=138.99 \AA, c=168.51 \AA$, and $\alpha=\beta=\gamma=90.00^{\circ}$. The collected data were commonly indexed, integrated, and scaled while using the HKL2000 suite [27]. Table 1 summarizes the statistics of the collected data.

\subsection{Structure Determination and Refinement}

The crystal structure was determined while using molecular replacement (MR) with the software Phaser-MR from PHENIX suite [28] using the crystal structure of sinapyl alcohol dehydrogenase from Populus tremuloides (PDB code: 1YQD) [17] as a search model. Model building was performed using the programs ARP/wARP [29] and Wincoot [30]. The refinement was performed using phenix.refine [31], and the final models of apo and complex had $R_{\text {work }}$ and $R_{\text {free }}$ values of $16.1 \%$ and $19.4 \%$, and $17.7 \%$ and $22.1 \%$, respectively. The geometric parameters of the final model were validated using WinCoot and MolProbity [32]. The experimental data of the structural models were assessed while using SFCHECK [33], and the refined models were deposited in the Protein Data Bank. Table 1 provides the refinement statistics.

\subsection{Docking Simulation}

The simulations of docking to the complex structure were performed using the Autodock Vina program [24]. The complex structure containing NADP and the fragment of PEG were used as a template. After removing the fragment of PEG, hydrogen atoms were added in accordance with only polar atoms. The structure of ligands were generated from the simplified molecular-input line-entry system and prepared as pdbqt files using CCP4MG (version 2.10.11, University of York, UK) [34] and Autodocking tools software (version 4.2, San Diego, CA, USA) [35]. The grid was set up with $x=20.52$, $y=41.94$, and $z=19.22$ at center_x $=65.40, y=59.95$, and $z=100.22$. An exhaustiveness value of eight was used for running software. The simulation results were checked while using PyMOL software (version 2.4, Schrödinger Inc., New York, USA).

\section{Conclusions}

In summary, we determined that the crystal structure of E. coli YjgB could be useful for designing a bio-synthetical pathway for the production of butanol, isobutanol, propanediol, butanediol, 2-PE, and 2-PEAc. We identified that a PEG fragment occupies the substrate binding pocket via $\pi-\pi$ stackings by NADP binding. Through docking simulation for the complex structure using four substrates, we suggested that $\pi-\pi$ stacking of aromatic rings in the active site is responsible for the broad substrate selectivity for several substrates.

Author Contributions: Cloning, protein purification, crystallization, G.T.N., J.-W.A.; Diffraction data collection and analysis, G.T.N., Y.-G.K., J.-W.A.; Docking simulation, J.-W.A.; Supervision, J.H.C.; Writing-original draft, 
G.T.N., J.-W.A., J.H.C.; Writing—review \& editing, G.T.N., J.-W.A., J.H.C. All authors have read and agreed to the published version of the manuscript.

Funding: This research was supported by Basic Science Research Program through the National Research Foundation of Korea funded by the Ministry of Science and ICT (grant No. NRF-2019R1A2C4069796 to JHC).

Acknowledgments: We would like to thank Jeong Eun Lee for the technical supports. We also thank beamline staff Sung Chul Ha at beamlines 7A of the Pohang Accelerator Laboratory (Pohang, Korea) for data collection.

Conflicts of Interest: The authors declare that they have no conflict of interest.

\section{References}

1. Atsumi, S.; Wu, T.-Y.; Eckl, E.-M.; Hawkins, S.D.; Buelter, T.; Liao, J.C. Engineering the isobutanol biosynthetic pathway in Escherichia coli by comparison of three aldehyde reductase/alcohol dehydrogenase genes. Appl. Microbiol. Biotechnol. 2010, 85, 651-657. [CrossRef]

2. Nakamura, C.E.; Whited, G.M. Metabolic engineering for the microbial production of 1,3-propanediol. Curr. Opin. Biotechnol. 2003, 14, 454-459. [CrossRef] [PubMed]

3. Berrios-Rivera, S.J.; San, K.Y.; Bennett, G.N. The effect of carbon sources and lactate dehydrogenase deletion on 1,2-propanediol production in Escherichia coli. J. Ind. Microbiol. Biotechnol. 2003, 30, 34-40. [CrossRef] [PubMed]

4. Yim, H.; Haselbeck, R.; Niu, W.; Pujol-Baxley, C.; Burgard, A.; Boldt, J.; Khandurina, J.; Trawick, J.D.; Osterhout, R.E.; Stephen, R.; et al. Metabolic engineering of Escherichia coli for direct production of 1,4-butanediol. Nat. Chem. Biol. 2011, 7, 445-452. [CrossRef] [PubMed]

5. Nielsen, D.R.; Yoon, S.-H.; Yuan, C.J.; Prather, K.L.J. Metabolic engineering of acetoin and meso-2,3-butanediol biosynthesis in E. Coli. Biotechnol. J. 2010, 5, 274-284. [CrossRef]

6. Heer, D.; Heine, D.; Sauer, U. Resistance of Saccharomyces cerevisiae to High. Concentrations of Furfural Is Based on NADPH-Dependent Reduction by at Least Two Oxireductases. Appl. Environ. Microbiol. 2009, 75, 7631-7638. [CrossRef]

7. Pick, A.; Rühmann, B.; Schmid, J.; Sieber, V. Novel CAD-like enzymes from Escherichia coli K-12 as additional tools in chemical production. Appl. Microbiol. Biotechnol. 2013, 97, 5815-5824. [CrossRef] [PubMed]

8. Jarboe, L.R. YqhD: a broad-substrate range aldehyde reductase with various applications in production of biorenewable fuels and chemicals. Appl. Microbiol. Biotechnol. 2011, 89, 249-257. [CrossRef]

9. Guo, D.; Zhang, L.; Pan, H.; Li, X. Metabolic engineering of Escherichia coli for production of 2-Phenylethylacetate from L-phenylalanine. Microbiologyopen 2017, 6, e00486. [CrossRef]

10. Guo, D.; Zhang, L.; Kong, S.; Liu, Z.; Li, X.; Pan, H. Metabolic Engineering of Escherichia coli for Production of 2-Phenylethanol and 2-Phenylethyl Acetate from Glucose. J. Agric. Food Chem. 2018, 66, 5886-5891. [CrossRef]

11. Kuo, C.H.; Chen, G.-J.; Chen, C.-I.; Liu, Y.-C.; Shieh, C.-J. Kinetics and optimization of lipase-catalyzed synthesis of rose fragrance 2-phenylethyl acetate through transesterification. Process. Biochem. 2014, 49, 437-444. [CrossRef]

12. Serra, S.; Fuganti, C.; Brenna, E. Biocatalytic preparation of natural flavours and fragrances. Trends Biotechnol. 2005, 23, 193-198. [CrossRef] [PubMed]

13. Brenna, E. Flavours and fragrances by biocatalytic routes. Agro. Food Ind. Hi-Tech. 2005, 16, $18-20$.

14. Sulzenbacher, G.; Alvarez, K.; van den Heuvel, R.H.H.; Versluis, C.; Spinelli, S.; Campanacci, V.; Valencia, C.; Cambillau, C.; Eklund, H.; Tegoni, M. Crystal structure of E. coli alcohol dehydrogenase YqhD: Evidence of a covalently modified NADP coenzyme. J. Mol. Biol. 2004, 342, 489-502. [CrossRef] [PubMed]

15. Persson, B.; Hedlund, J.; Jörnvall, H. Medium-and short-chain dehydrogenase/reductase gene and protein families: The MDR superfamily. Cell. Mol. Life Sci. 2008, 65, 3879. [CrossRef]

16. Nordling, E.; Jörnvall, H.; Persson, B. Medium-chain dehydrogenases/reductases (MDR) Family characterizations including genome comparisons and active site modelling. Eur. J. Biochem. 2002, 269, 4267-4276. [CrossRef]

17. Jun, S.Y.; Walker, A.M.; Kim, H.; Ralph, J.; Vermerris, W.; Sattler, S.E.; Kang, C.H. The Enzyme Activity and Substrate Specificity of Two Major Cinnamyl Alcohol Dehydrogenases in Sorghum (Sorghum bicolor), $\mathrm{SbCAD}_{2}$ and $\mathrm{SbCAD}_{4}$. Plant. Physiol. 2017, 174, 2128-2145. [CrossRef]

18. Rao, S.T.; Rossmann, M.G. Comparison of super-secondary structures in proteins. J. Mol. Biol. 1973, 76, $241-256$. [CrossRef] 
19. Krissinel, E.; Henrick, K. Inference of macromolecular assemblies from crystalline state. J. Mol. Biol. 2007, 372, 774-797. [CrossRef]

20. Thompson, J.D.; Higgins, D.G.; Gibson, T.J. CLUSTAL W: Improving the sensitivity of progressive multiple sequence alignment through sequence weighting, position-specific gap penalties and weight matrix choice. Nucleic Acids Res. 1994, 22, 4673-4680. [CrossRef]

21. Gouet, P.; Courcelle, E.; Stuart, D.I.; Metoz, F. ESPript: Analysis of multiple sequence alignments in PostScript. Bioinformatics 1999, 15, 305-308. [CrossRef] [PubMed]

22. Raj, S.B.; Ramaswamy, S.; Plapp, B.V. Yeast Alcohol Dehydrogenase Structure and Catalysis. Biochemistry 2014, 53, 5791-5803. [CrossRef] [PubMed]

23. Thomas, L.M.; Harper, A.R.; Miner, W.A.; Ajufo, H.O.; Branscum, K.M.; Kao, L.; Sims, P.A. Structure of Escherichia coli AdhP (ethanol-inducible dehydrogenase) with bound NAD. Acta Cryst. Sect. F-Struct. Biol. Cryst. Commun. 2013, 69, 730-732. [CrossRef] [PubMed]

24. Trott, O.; Olson, A.J. AutoDock Vina: Improving the speed and accuracy of docking with a new scoring function, efficient optimization, and multithreading. J. Comput. Chem. 2010, 31, 455-461. [CrossRef] [PubMed]

25. McQueen, C. Comprehensive Toxicology (Second Edition); Elsevier: Amsterdam, The Netherlands, 2017; pp. $291-330$.

26. Singh, S.; Brocker, C.; Koppaka, V.; Chen, Y.; Jackson, B.C.; Matsumoto, A.; Thompson, D.C.; Vasiliou, V. Aldehyde dehydrogenases in cellular responses to oxidative/electrophilic stress. Free Radic. Biol. Med. 2013, 56, 89-101. [CrossRef]

27. Otwinowski, Z.; Minor, W. Processing of X-ray diffraction data collected in oscillation mode. Methods Enzym. 1997, 276, 307-326.

28. Zwart, P.H.; Afonine, P.V.; Grosse-Kunstleve, R.W.; Hung, L.W.; Ioerger, T.R.; McCoy, A.J.; McKee, E.; Moriarty, N.W.; Read, R.J.; Sacchettini, J.C.; et al. Automated structure solution with the PHENIX suite. Methods Mol. Biol. 2008, 426, 419-435.

29. Langer, G.; Cohen, S.X.; Lamzin, V.S.; Perrakis, A. Automated macromolecular model building for X-ray crystallography using ARP/wARP version 7. Nat. Protoc. 2008, 3, 1171-1179. [CrossRef] [PubMed]

30. Emsley, P.; Cowtan, K. Coot: Model-building tools for molecular graphics. Acta Cryst. D-Biol. Cryst. 2004, 60, $2126-2132$. [CrossRef]

31. Afonine, P.V.; Grosse-Kunstleve, R.W.; Echols, N.; Headd, J.J.; Moriarty, N.W.; Mustyakimov, M.; Terwilliger, T.C.; Urzhumtsev, A.; Zwart, P.H.; Adams, P.D. Towards automated crystallographic structure refinement with phenix.refine. Acta Cryst. D-Biol. Cryst. 2012, 68, 352-367. [CrossRef]

32. Chen, V.B.; Arendall, W.B.; Headd, J.J.; Keedy, D.A.; Immormino, R.M.; Kapral, G.J.; Murray, L.W.; Richardson, J.S.; Richardson, D.C. MolProbity: All-atom structure validation for macromolecular crystallography. Acta Crystallogr. Sect. D-Biol. Crystallogr. 2010, 66, 12-21. [CrossRef] [PubMed]

33. Vaguine, A.A.; Richelle, J.; Wodak, S.J. SFCHECK: a unified set of procedures for evaluating the quality of macromolecular structure-factor data and their agreement with the atomic model. Acta Crystallogr. Sect. D-Biol. Crystallogr. 1999, 55, 191-205. [CrossRef] [PubMed]

34. McNicholas, S.; Potterton, E.; Wilson, K.S.; Noble, M.E. Presenting your structures: The CCP4mg molecular-graphics software. Acta Crystallogr. D-Biol. Crystallogr. 2011, 67, 386-394. [CrossRef] [PubMed]

35. Morris, G.M.; Huey, R.; Lindstrom, W.; Sanner, M.F.; Belew, R.K.; Goodsell, D.S.; Olson, A.J. AutoDock4 and AutoDockTools4: Automated docking with selective receptor flexibility. J. Comput. Chem. 2009, 30, $2785-2791$. [CrossRef] [PubMed]

Sample Availability: Samples of are not available from the authors. 